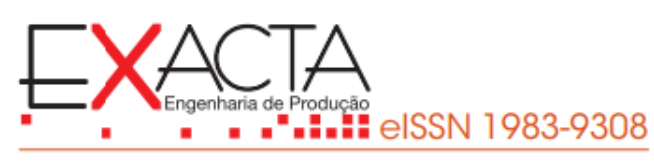

\title{
DESENVOLVIMENTO DE UM MODELO FUZZY MULTICRITÉRIO PARA ANÁLISE DE CRITICIDADE: O CASO DO TREM DE LEVITAÇÃO MAGNÉTICA
}

\author{
DEVELOPMENT OF A MULTICRITERIA FUZZY MODEL FOR CRITICALITY \\ ANALYSIS: THE CASE OF THE MAGNETIC LEVITATION TRAIN
}

Versão do autor aceita publicada online: 24 jun. 2019

Publicado online: 12 maio 2021

Como citar esse artigo - American Psychological Association (APA):

Sucena, M. P., Aprigio, T. V., Peres, M. D. da C., Cunha, C. P., \& Santos, J. E. (2021, abr./jun.). Desenvolvimento de um modelo fuzzy multicritério para análise de criticidade: o caso do trem de levitação magnética. Exacta, 19(2), 412-431. https://doi.org/10.5585/exactaep.2021.10333.

Submeta seu artigo para este periódico $\beta$

Dados Crossmark 


\title{
DESENVOLVIMENTO DE UM MODELO FUZZY MULTICRITÉRIO PARA ANÁLISE DE CRITICIDADE: O CASO DO TREM DE LEVITAÇÃO MAGNÉTICA
}

\author{
DEVELOPMENT OF A MULTICRITERIA FUZZY MODEL FOR CRITICALITY ANALYSIS: \\ THE CASE OF THE MAGNETIC LEVITATION TRAIN
}

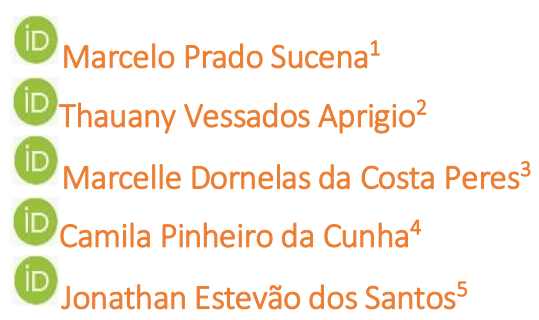

1 Doutor

Fundação Getulio Vargas - FGV. marcelo.sucena@fgv.br

2 Engenheira de Produção Universidade Estácio de Sá thauany.aprigio@gmail.com

${ }^{3}$ Engenheira de Produção Universidade Estácio de Sá marcelle.dc@gmail.com

${ }^{4}$ Engenheira de Produção Universidade Estácio de Sá camila.pinheiro94@yahoo.com.br

${ }^{5}$ Engenheiro de Produção Universidade Estácio de Sá johnsantos.03@hotmail.com

Recebido em: 23 jan. 2019

Aprovado em: 24 jun. 2019
Resumo: Este artigo objetiva apresentar o desenvolvimento de um modelo matemático multicritério, utilizando o método F-SAW, híbrido entre a Teoria Fuzzy e o método multicritério SAW (Simple Additive Weighting), para determinar a criticidade dos elementos que compõem um sistema complexo. Utilizou-se, inicialmente, a pesquisa exploratória bibliográfica pela necessidade de se obter melhor conhecimento sobre os critérios a serem empregados no modelo para avaliação da criticidade. Identificaram-se 182 critérios que, após triagem, foram selecionados 25. Quanto ao estudo de caso, que objetiva exemplificar e validar os procedimentos do modelo, desenvolveu-se a pesquisa descritiva como base para a coleta de dados sobre as partes que compõem o sistema complexo estudado, o MagLev-Cobra, desenvolvido na COPPE/UFRJ. De posse dos critérios e das 53 partes, desenvolve-se o modelo matemático onde é realizado o processamento dos dados. Como resultado, foi possível gerar uma lista hierarquizada pelo índice de criticidade dos componentes do sistema complexo. Desta forma, percebeu-se que o elemento com maior índice de criticidade é o Criostato; em contrapartida, os menos críticos foram o voltímetro e amperímetro. Nota-se que o modelo gerado é de ampla aplicação em sistemas complexos de quaisquer áreas, haja vista que os critérios utilizados são genéricos. Além disso, para a fase de certificação que o sistema está submetido, a existência de um modelo preciso, que considere vasta abordagem de critérios, denota maior confiança nos resultados da produção com segurança.

Palavras-chave: Criticidade. Análise multicritério. Fuzzy. SAW. MagLev-Cobra.

Abstract: This article aims to generate a multicriteria mathematical model, using the F-SAW method, hybrid between Fuzzy Theory and the Simple Additive Weighting (SAW) method, to determine the criticality of the elements that make up a complex system. It was used, initially, an exploratory bibliographical research on the need for a greater knowledge on the latest data and a model for critical evaluation. 182 criteria were identified that, after screening, were selected 25 . As for the case study, which aims to exemplify and validate the procedures of the model, the descriptive research was developed as the basis for collecting data on the parts that make up the complex system, the MagLev-Cobra, developed at COPPE/UFRJ. With the criteria and 53 parts, the mathematical model is developed where the data processing is performed. As a result, it was possible to generate a hierarchical list by the criticality index of the components of the complex system. In this way, the component that needs greatest attention in maintenance activities is the Cryostat; in contrast, the least critical were the Voltmeter and Ammeter. It should be noted that the generated model can be used in complex systems of any areas, since the criteria used are generic. In addition, for the certification phase the system is undergoing, the existence of a precise model, which considers a broad approach of criteria, indicates greater confidence in the results of production with safety.

Keywords: Criticality. Multicriteria analysis. Fuzzy. SAW. MagLev-Cobra. 


\section{Considerações iniciais}

No atual cenário industrial, devido à alta competitividade existente entre as organizações e o contínuo esforço pelo aumento da produtividade, é cada vez mais intensa a busca pela redução das falhas que podem ocorrer em sistemas, sejam eles complexos ou não, pertencentes a um sistema produtivo, podendo gerar gargalos, interferindo na produção e prejudicando as metas e objetivos da empresa.

Entretanto, com os constantes e rápidos avanços tecnológicos é comum nas empresas a presença de sistemas complexos de alta tecnologia, permitindo a automação e integração de determinados processos, entretanto, que geram situações de riscos de falhas, que são inevitáveis. Por esse motivo, a manutenção tornou-se um dos fatores chave para o sucesso industrial, pois o que antes só era visto como uma medida corretiva, hoje está diretamente associada à disponibilidade e confiabilidade dos insumos e produtos finais.

Mas, na verdade, como os recursos para execução da manutenção são limitados, faz-se necessário identificar os elementos que podem interferir mais severamente em um sistema produtivo pela determinação das suas criticidades.

Nesse contexto, para garantir a segurança, disponibilidade e confiabilidade dos componentes de certo sistema é necessário conhecer e analisar cada risco de falha existente neles, além das suas respectivas criticidades, para que, dessa forma, a produção extraia o máximo de rendimento possível.

Assim, nesse artigo pretende-se desenvolver um modelo matemático multicritério, utilizando o método F-SAW, para se determinar a criticidade dos elementos que compõem um sistema complexo. Tal modelo será aplicado, para exemplificar e validá-lo, ao sistema complexo MagLev-Cobra, trem de levitação magnética desenvolvido na COPPE/UFRJ.

Atualmente o sistema MagLev-Cobra não conta com um método que indique os seus elementos mais críticos e que forneça uma lista hierarquizada, por intermédio de índice de criticidade dos componentes do sistema. A existência desse método permitirá que o Planejamento e o Controle da Manutenção (PCM) seja mais eficiente quanto à alocação de recursos.

O modelo desenvolvido proporcionará a identificação dos elementos mais necessitados de atenção, inclusive para alocação de recursos, uma vez que esses são limitados e devem ser aplicados prioritariamente nas partes que impliquem na execução das funções do MagLev-Cobra.

A fim de avaliar os critérios de forma qualitativa e quantitativa, será utilizado o método F-SAW, que é a união do método multicritério SAW (Simple Additive Weighting) com o uso de números Fuzzy. Essa união permite o tratamento de valores lineares e não lineares, com dimensões diferentes e, principalmente, valores vagos. Deste modo, a sua utilização proporcionará melhor tratamento da subjetividade obtida nas avaliações dos especialistas do sistema em estudo. 


\section{Sustentação teórica}

\subsection{Análise da criticidade}

As normas MIL-STD-1629A (1980) e BS5760 (1991) definem "criticidade" como "uma medida relativa das consequências e a frequência de ocorrência de falhas". Também definem "análise de criticidade" como "um procedimento para listar modos de falhar de forma hierarquizada, combinando a severidade e a probabilidade de ocorrência". Ou seja, a análise de criticidade permite ranquear os componentes de um sistema, de acordo com a sua criticidade, de modo a avaliar qual deles é o mais crítico. Com isso, é possível tomar providências preventivas, a fim de, caso ocorra uma falha, evitar gargalos na produção e aumentar a disponibilidade e confiabilidade dos ativos.

Nesse contexto, a análise de criticidade assume um papel importante, pois é possível avaliar quais são os elementos mais críticos do sistema produtivo (sejam eles equipamentos, componentes de equipamentos ou até mesmo processos) para que sejam priorizados no processo de investimento.

\subsection{F-Saw (Fuzzy - Simple Additive Weighting)}

Como um dos instrumentos para apoio à decisão tem-se a Análise Multicritério (AM). "Os métodos de apoio multicritério à decisão (ou métodos de análise multicritério) começaram a surgir a partir da década de 1970" (Gomes, Araya, \& Carignano, 2004 como citado em Leal, 2010, p. 67) e são utilizados para auxiliar na escolha da alternativa mais adequada quando é preciso avaliar, simultaneamente, uma multiplicidade de critérios que muitas vezes são conflitantes entre si.

Segundo Nakajima, Da Silva e Miranda (2007), "métodos de modelagem multicritério são projetados para encontrar soluções alternativas preferenciais, de acordo com sua adaptação para o problema. Esses métodos investigam alternativas utilizando critérios e prioridades múltiplas".

São vantagens da abordagem multicritério: "a constituição de uma base de diálogo, utilizando diversos pontos de vista comuns; maior facilidade para incorporar incertezas; enfrentar cada solução como um compromisso de objetivos em conflito" (Gomes et al., 2004 como citado em Leal, 2010, p. 67).

O método SAW é um dos mais conhecidos e amplamente utilizado devido a sua simplicidade. Nessa técnica, as alternativas e os critérios são agrupados em uma matriz de decisão e, em seguida, é realizada uma sequência de passos a fim de se encontrar a alternativa mais adequada. Ele permite um ranqueamento das alternativas, possibilitando a geração de uma lista hierarquizada da melhor alternativa para a pior, ou vice-versa.

O processo consiste em quantificar os valores dos atributos (critérios) para cada alternativa, construindo a matriz de decisão contendo estes valores, derivando a matriz de decisão normalizada, nomeando a importância (pesos) para os critérios e calculando a contagem global para cada alternativa. 
A alternativa com a contagem mais alta é selecionada como a melhor. (Janic \& Reggiani, 2002 como citado em Rossoni \& Meireles, 2011, p. 3).

"Sua aplicação prática pode enfrentar limitações inerentes ao processo por conter imprecisões quanto aos dados coletados, como por exemplo, quando há a necessidade de expressar certos critérios de forma qualitativa" (Sucena, 2017). Por este motivo, como tratativa às limitações inerentes a coleta de dados imprecisos, serão utilizados os números Fuzzy redundando então no método híbrido denominado F-SAW.

O método F-SAW (Fuzzy Simple Additive Weighting) pode ser utilizado tanto em decisões individuais como em decisões em grupo. Segundo Chou, Chang e Shen (2008), "em uma configuração de grupo, avaliações com números Fuzzy podem ser agregadas por vários métodos". "Entre eles, cinco são mais populares, incluindo média, mediana, máximo, mínimo e operadores mistos" (Buckley, 1984 as cited in Chou et al., 2008, p. 137).

"Embora o operador médio seja o método de agregação mais utilizado" (Liang \& Wang, 1991 as cited in Chou et al., 2008, p. 137), a importância (ou a confiabilidade) dos decisores individuais (ou especialistas) pode ser desigual na prática. Isso se dá porque, às vezes, há decisores que são mais importantes ou mais confiáveis que outros no grupo de decisão.

\subsection{Números fuzzy}

"Também conhecida como Teoria dos Conjuntos Difusos ou Nebulosos, a teoria dos conjuntos Fuzzy surgiu em 1965 com a publicação do trabalho do professor Lofti A. Zadeh" (De Almeida, Moré, \& Villela, 2010), intitulado "Fuzzy Sets", na revista "Information and Control". Essa teoria, diferentemente da lógica Aristotélica, permite considerar que uma asserção seja não totalmente verdadeira e, ao mesmo tempo, não totalmente falsa. Em outras palavras, a Teoria Fuzzy pode ser vista como uma teoria matemática para a representação de incertezas.

Um conjunto Fuzzy pertencente a um intervalo numérico mensurável ou universo de discurso $(U)$, que é definido por uma função de pertinência que relaciona os valores de $U$ à valores de um intervalo que varia entre 0 e 1 , valores também chamados de graus de pertinência $(\mu \mathrm{A})$, que indicam o quanto um determinado elemento pertence ou não a esse conjunto.

Se o grau de pertinência de um elemento é 0 , significa que o mesmo não pertence ao conjunto; e se o grau de pertinência é 1 , significa que o elemento pertence totalmente ao conjunto.

Os termos linguísticos são nomes (p.e. frio, baixo, iluminado, caro etc.) que identificam as funções de pertinência, geralmente, adjetivos ou advérbios que, segundo Gomide, Gudwin e Tanscheit (1995 como citado em Brum, Do Carmo, \& Moreira, 2017, p. 11), "representam partes de certa variável Fuzzy". 
As funções de pertinência mais utilizadas são as triangulares e as trapezoidais, uma vez que elas possuem maior simplicidade na implementação computacional. Segundo Souza (1999 como citado em Sucena, 2017, p. 12), essas funções podem ser representadas da seguinte forma:

$\checkmark$ Triangular - é descrita pelas variáveis SL, C e SR (Spread Left, Center e Spread Right, respectivamente), onde:

$$
\mu(x)=\left\{\begin{array}{c}
\mu(\mathrm{x})=\frac{\mathrm{x}-\mathrm{SL}}{\mathrm{C}-\mathrm{SL}}, \text { para } \mathrm{SL} \leq \mathrm{x} \leq \mathrm{C} ; \\
\mu(\mathrm{x})=\frac{\mathrm{x}-\mathrm{SR}}{\mathrm{C}-\mathrm{SR}}, \text { para } \mathrm{C} \leq \mathrm{x} \leq \mathrm{SR} ; \mathrm{e} \\
\mu(\mathrm{x})=0, \text { caso contrário. }
\end{array}\right.
$$

$\checkmark$ Trapezoidal - é descrita pelas variáveis a, b, c e d que regulam a inclinação das bordas, onde:

$$
\mu(x)=\left\{\begin{aligned}
0, & x \leq a \\
1-\frac{b-x}{b-a}, & a<x \leq b \\
1, & b<x \leq c \\
1-\frac{d-x}{d-c}, & c<x \leq d \\
0, & x>d
\end{aligned}\right.
$$

Um conjunto Fuzzy A, convexo, normalizado, definido em $\mathfrak{R}$, tal que exista pelo menos um $\mathrm{x}$ com relação a $\mu \mathrm{A}(\mathrm{x})=1$ e que $\mu \mathrm{A}$ seja contínua por partes, é denominado Número Fuzzy e são utilizados para quantificar atributos que tenham imprecisão na forma de expressar. (Sucena, 2017).

Onde $\mu \mathrm{A}(\mathrm{x})$ representa o grau de pertinência de um determinado elemento $x$ pertencente ao universo de discurso $U$ e $\mu A$ a função de pertinência.

"Existe uma grande variedade de tipos de números Fuzzy" (Kaufmann \& Gupta, 1988 como citado em Montevechi \& De Pinho, 2017, p. 4), entretanto, "os mais usados são os triangulares e trapezoidais" (Sucena, 2017), tendo em vista que esses são mais simples e de fácil processamento computacional.

Um número Fuzzy triangular pode ser definido como $A=(S L, C, S R)$, conforme a expressão 1. Um número Fuzzy trapezoidal pode ser definido como $A=(a, b, c, d)$, de acordo com a expressão 2.

Para o número triangular o valor $C$, pertencente ao universo de discurso $U$, é atribuído um grau de pertinência 1 , ou seja, há uma grande possibilidade de que esse valor ocorra. Aos valores intermediários, entre $S L$ e $C$ e entre $C$ e $S R$, são atribuídos valores de pertinência entre 0 e 1 . Já aos valores menores que SL e maiores que SR são atribuídos graus de pertinência zero, ou seja, não há a possibilidade de que esses valores ocorram. 
Para o número trapezoidal a abordagem é a mesma que a anterior, exceto que há infinitos valores centrais, onde se admitem graus de pertinência iguais a 1. Esses valores estão restritos à faixa entre $b$ e $c$ do universo de discurso $U$.

É possível realizar operações aritméticas com números Fuzzy, tais como adição, subtração, produto, divisão e produto de um escalar. Utilizando-se dois números triangulares Fuzzy A1 = $(a 11, a 12$, a13) e $A 2=(a 21, a 22, a 23)$ e transformando-os em números trapezoidais, tal que $A 1=(a 11, a 12, a 12$, a13) e A2 = (a21, a22, a22, a23), têm-se as seguintes operações:

$$
\begin{aligned}
& \checkmark \text { Adição: } A 1+A 2=(a 11+a 21, a 12+a 22, a 12+a 22, a 13+a 23) \text {; } \\
& \checkmark \quad \text { Subtração: } A 1-A 2=(\text { a11 - a23, a12 - a22, a12 - a22, a13- a21); } \\
& \checkmark \quad \text { Produto: } \mathrm{A} 1 \times \mathrm{A2}=(\mathrm{a} 11 \times \mathrm{a} 21, \mathrm{a} 12 \times \mathrm{a} 22, \mathrm{a} 12 \times \mathrm{a} 22, \mathrm{a} 13 \times \mathrm{a} 23) \text {; } \\
& \checkmark \text { Divisão: A1 / A2 = (a11 /a23, a12 / a22, a12 / a22, a13/ a21); } \\
& \checkmark \quad \text { Produto de um escalar } n: n . \mathrm{A} 1=(n \times \mathrm{a} 11, n \times \mathrm{a} 12, n \times \mathrm{a} 12, n \times \mathrm{a} 13) \text {. }
\end{aligned}
$$

Para se converter um número triangular ou trapezoidal Fuzzy em um número Real pode ser utilizada a distribuição PERT, conforme as expressões 8 e 9 adiante.

$$
R=\frac{A 1+4 A 2+A 3}{6}
$$

Em que,

A1: valor mínimo;

A2: valor mais provável;

$\mathrm{A} 3=$ valor máximo .

$$
R=\frac{A 1+4 \frac{(A 2+A 3)}{2}+A 4}{6}
$$

Em que,

A1: valor mínimo;

A2: valor inferior mais provável;

A3: valor superior mais provável;

A4: valor máximo. 


\section{Procedimentos metodológicos}

\subsection{Objeto de estudo}

"O MagLev-Cobra é um veículo de levitação magnética em desenvolvimento no LASUPCOPPE/UFRJ. É um sistema de transporte, energeticamente eficaz, que não gera ruído, vibrações, emissões gasosas e tem implantação mais econômica do que outros sistemas ferroviários similares e tradicionais" (Sucena \& Stephan, 2012).

O seu funcionamento baseia-se nas características da Levitação Supercondutora, isto é, na propriedade diamagnética dos supercondutores para exclusão do campo magnético do interior dos supercondutores. Uma placa de cerâmica supercondutora ao ser resfriada com nitrogênio líquido, produz o efeito de levitação sobre um ímã de terras raras, ou seja, basta resfriar o supercondutor que imediatamente se dá a levitação.

O MagLev-Cobra é acionado por motor elétrico de indução linear síncrono (LSM). É um motor eficiente que funciona com movimento longitudinal ao invés de rotação e constitui o principal componente para motorização de um trem de levitação magnética, que por concepção, não possui contato com a superfície de rolamento. (Sucena, 2012).

O MagLev-Cobra foi selecionado pois é um sistema complexo que não conta com um método que indique os seus elementos mais críticos e que forneça uma lista hierarquizada, por intermédio de índice de criticidade dos componentes do sistema.

O estudo de Costa (2017) expõe o veículo do Sistema MagLev-Cobra dividido em três níveis de detalhamento:

Nível 1 (O sistema: veículo MagLev-Cobra); Nível 2 (Os subsistemas: carroceria, porta veicular, controle veicular, monitoramento, tração/potência, freio mecânico e levitação e abastecimento a vácuo); Nível 3 (Componentes dos subsistemas).

Os componentes do Nível 3, os mais baixos na hierarquia do sistema e, portanto, mais operacionais, serão utilizados no modelo matemático. São eles: Carroceria (Painel de equipamentos, Suporte de equipamentos, Conexão entre vagões, Banco, Pega mão, Piso, Vedação entre vagões, Estrutura de fixação dos Criostatos e Estrutura de fixação das rodas de segurança); Porta veícular (Folha de porta, Motor DC, Eixo árvore, Barra estabilizadora, Rolamentos, Placa de controle, Fim de Curso, Fuso, Castanha, Guia e Espaçador); Controle veicular (Alavanca de comando, CLP TPW, IHM e Ladder); Monitoramento (Sensor de altura, Sensor de velocidade, Sensor de contagem de barras, Câmera veicular, Acelerômetro, Amperímetro, Voltímetro, Medidor de freq. do inversor, Equipamento de aquisição de dados, Software de Proc. Dados e HD); Tração/potência (Coletores, Primário do motor linear, Inversor de frequência e Disjuntor); Freio mecânico (Bomba hidráulica, Tanque de óleo, Acumulador e Mangotes flexíveis); Terminal de mangote, Manômetro, Pistão esquerdo, Pistão direito e 
Sapata de freio; Botão de acionamento no painel e Pressostato do acumulador; Levitação (Criostato e Fixação do criostato); Abastecimento e vácuo (Fittings de vácuo).

\subsection{Procedimentos de coleta de dados}

Inicialmente, foram revisados 46 artigos científicos para identificação dos critérios a serem utilizados no modelo matemático, totalizando 182 possíveis critérios. Estes foram agrupados e confrontados com os autores onde foram citados, gerando-se o cálculo da frequência dessas citações.

Os 46 artigos são: MIL-STD-1629A (1980), BS5760 (1991), Bowles e Peláez (1995), Teng e Ho (1996), Chareonsuk, Nagarur, e Tabucanon (1997), Noronha (1998), Pamplona (1999), Andrade e Turrioni (2000), De Abreu, Granemann, Gartner e Bernardes (2000), De Almeida (2001), Sucena (2002), Xu, Tang, Xie, HO e Zhu (2002), De Almeida (2004), Cavalcante e De Almeida (2005), Hijes e Cartagena (2006), Moteff (2005), Ben (2006), Ferraboli (2006), Leal, De Pinho e De Almeida (2006), Szajubok, Mota, e De Almeida (2006), Cavalcante, Ferreira, Brito, e De Almeida (2007), Cavalcante e De Almeida (2007), Helman e Marçal (2007), Chou et al. (2008), Da Silva e Belderrain (2009), Ilangkumaran e Kumanan (2009), Lins (2009), Marins Marins, Souza e Barros (2009), Moreira (2009), Prado, Souza e Yoshizaki (2009), Sachdeva, Kuma e Kumar (2009), Sardinha, Claro e Pereira (2009), Lins, Garcez e De Almeida (2011), Macedo (2011), De Oliveira, Alencar e Costa (2012), MIL-STD-882E (2012), Shaghaghi e Rezaieb (2012), Zammori e Gabbrielli (2012), Azizi e Fathi (2014), Jaderi, Ibrahim, Jaafarzadeh, Abdullah, Yavari, Shamsudin, e Nabavi. (2014), Baran (2015), Bueno, Ikuno, De Araújo, Lima, Moreira e De Melo (2015), Secretaria de Logística e Tecnologia da Informação Secretaria de Logística e Tecnologia da Informação [SLTI/MPOG], Centro de Tecnologia da Informação Renato Archer do Ministério da Ciência, Tecnologia e Inovação [CTI/MCTI] e Fundação de Apoio à Capacitação em Tecnologia da Informação [FACTI] (2015), Olsen (2015), Cez e Farias (2016), Mierau (2017) e Mitre: Systems Engineering Guide (2017).

Foram então selecionados 25 critérios para utilização no modelo matemático. Tal seleção foi baseada na retirada de redundâncias, no agrupamento de critérios iguais com títulos diferentes, na opção por maiores frequências de citações e na coerência com a análise da criticidade de sistemas complexos.

Os critérios elegidos, assim como as suas descrições, são os seguintes:

A. Confiabilidade: Qual é a chance de que uma falha não ocorra no componente, em um determinado instante de tempo e sob condições predeterminadas?

B. Confidencialidade: Qual a garantia de que a informação sobre a falha no componente seja legível somente para pessoas autorizadas? 
C. Correlação: Qual o grau de relação do componente em questão com a principal função do sistema?

D. Custo da atividade manutenção: Qual o nível de interferência desse componente no custo médio dos serviços de manutenção gastos em caso de falha?

E. Custo de peças sobressalentes: Caso exista uma falha nesse componente, qual seria o custo de se ter peças em estoque para possíveis substituições?

F. Custo da atividade substituição: Caso exista uma falha nesse componente, quais são os custos específicos, inclusive os de mão de obra, relacionados à atividade da sua substituição?

G. Custo de aquisição do componente: Para concepção de sistema novo esse componente contribui de que forma no custo total do sistema?

H. Custo esperado das consequências das falhas para o sistema: Caso ocorra uma falha nesse componente, qual é a contribuição para os custos de mitigação dos efeitos negativos que derivam dessas falhas?

I. Custo da atividade operacional: Na ocorrência de uma falha nesse componente, como são as influências nos custos operacionais e de manutenção do sistema?

J. Custos da parada de produção: Ocorrendo uma falha nesse componente, como será a interferência na visão da demanda quanto a não produção?

K. Dano ambiental: Na ocorrência de uma falha nesse componente, qual será a influência nos prejuízos ao meio ambiente, considerando a hipótese de indenização a terceiros?

L. Dano financeiro: Na ocorrência de uma falha nesse componente, qual será a influência nos custos administrativos?

M. Dano humano: Na ocorrência de uma falha nesse componente, qual será a influência nos prejuízos às pessoas?

N. Dano operacional: Na ocorrência de uma falha nesse componente, qual será a influência na operação do sistema?

O. Detecção: É possível se detectar uma falha nesse componente antes que ela afete a função principal do sistema?

P. Dificuldade de reparo: Caso ocorra uma falha nesse componente, qual será a complexidade para repará-lo ou substituí-lo?

Q. Disponibilidade de fornecedores: Há disponibilidade de fornecedores qualificados no mercado, caso ocorra a necessidade de aquisição desse componente?

R. Disponibilidade de peças sobressalentes: Há necessidade de se ter peças de reposição para manutenção desse componente, considerando a sua acessibilidade no comércio? 
S. Fator de velocidade de manifestação da falha: Qual é o tempo que pode transcorrer entre o momento em que se detecta uma falha em potencial no componente e o momento em que esta se transforma em falha funcional do sistema?

T. Idade do equipamento: Qual é a relação do tempo de vida do componente com a possibilidade de ocorrência de falhas?

U. Localização: Para manutenção, esse componente é de fácil acesso no sistema?

V. Mantenabilidade: Caso ocorra uma falha, qual é o tempo de recuperação do componente, ou seja, qual é o tempo para voltar ao seu estado operacional?

W. Maturidade técnica: Há maturidade das tecnologias associadas a esse componente?

X. Segurança: Qual é o impacto das falhas sobre a segurança do sistema, das pessoas e do meioambiente?

Y. Vulnerabilidade: Há interferência das condições ambientais no funcionamento do componente que afetem as funções do sistema?

Para preenchimento da matriz de decisão (relação Componente $x$ Critério) foram consultados especialistas do Laboratório de Aplicações de Supercondutores (LASUP), do Programa de Engenharia Elétrica da COPPE/UFRJ, onde está em desenvolvimento o objeto do estudo de caso.

Cada especialista registrou a sua opinião, de forma qualitativa, quanto ao impacto de cada componente na função transporte do MagLev-Cobra, segundo cada critério usando os Termos Linguísticos apresentados na Tabela 1 a seguir. 
Tabela 1 - Síntese dos critérios

Critérios

(A) Confiabilidade

(B) Confidencialidade

(C) Correlação

(D) Custo da atividade manutenção

(E) Custo de peças sobressalentes

(F) Custo da atividade substituição

(G) Custo de aquisição do ativo

(H) Custo esperado das consequências das falhas para o sistema

(I) Custo da atividade operacional

(J) Custos da parada de produção

(K) Dano ambiental

(L) Dano financeiro

(M) Dano humano

(N) Dano operacional

(O) Deteç̧ão

(P) Dificuldade de reparo

(Q) Disponibilidade de fornecedores

(R) Disponibilidade de peças sobressalentes

(S) Fator de velocidade de manifestação da falha

(T) Idade do equipamento

(U) Localização

(V) Mantenabilidade

(W) Maturidade técnica

(X) Segurança

(Y) Vulnerabilidade
Termos Linguísticos 1

Alta

Alta

Fraco

Baixo

Baixo

Baixo

Barato

Baixo

Baixo

Baixo

Pequeno

Pequeno

Pequeno

Pequeno

Fácil

Pouca

Alta

Muito Acessível

Lento

Novo

Muito Acessível

Baixa

Alta

Insignificante

Pouco Vulnerável Razoável

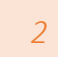

Média

Baixa

Média

Baixa

Moderado Forte

Médio

Médio

Médio

Médio

Médio

Médio

Médio

Médio

Médio

Médio

Médio

Regular

Razoável

Média

Elevado

Elevado

Elevado

Caro

Elevado

Elevado

Elevado

Grande

Grande

Grande

Grande

Difícil

Muita

Baixa

Pouco Acessível

Razoável Rápido

Intermediário Velho

Acessível Pouco Acessível

Média Alta

Média Baixa

Razoável Significativo

Muito Vulnerável

Fonte: Próprios Autores.

\subsection{Procedimentos de análise de dados}

Para o processamento dos dados, o modelo matemático foi implementado em planilha eletrônica Microsoft Excel.

As opiniões dos especialistas foram então convertidas para números Fuzzy. Cada termo linguístico que representa a relação Critério x Componente, utilizado na matriz de decisão, foi representado por números Fuzzy triangulares e/ou trapezoidais.

Esta representação se dará usando as seguintes variáveis Fuzzy expressas pelas suas integrais-

Fuzzy, conforme as expressões 10, 11 e 12.

$$
\begin{aligned}
\text { Termo linguístico } 1=\int_{0 \rightarrow 1}^{1 \rightarrow 1} 1 / x+\int_{1 \rightarrow 1}^{4 \rightarrow 0} \frac{x-4}{-3} / x \\
\text { Termo linguístico } 2=\int_{3 \rightarrow 0}^{5 \rightarrow 1} \frac{x-3}{2} / x+\int_{5 \rightarrow 1}^{7 \rightarrow 0} \frac{x-7}{-2} / x \\
\text { Termo linguístico } 3=\int_{6 \rightarrow 0}^{10 \rightarrow 1} \frac{x-6}{4} / x
\end{aligned}
$$


Por se entender que os dados coletados resultarão de vários especialistas, será necessário obter um valor único que represente o consenso deles. Dessa forma, será aplicada a média aritmética Fuzzy a todas as respostas dos especialistas, conforme as expressões 3 e 6 .

Para se tratar cada relação Critério x Componente faz-se necessário converter os números Fuzzy Agregados em números Crisp que são valores Reais ( $\Re)$. Essa conversão será efetuada pela distribuição PERT, com os números Fuzzy triangulares e/ou trapezoidais de cada especialista, conforme as expressões 8 e 9.

Cada critério tem um peso na avaliação da criticidade e, consequentemente, no modelo matemático. Esses pesos serão qualificados usando os termos linguísticos "baixa, média e alta" para identificar a importância desse critério para avaliação da criticidade. A coleta de dados se dará segundo a visão dos mesmos especialistas que avaliaram as relações Critérios $\times$ Componentes, de forma qualitativa.

Segundo Sucena (2017), "a criticidade é obtida por meio da expressão 9, que representa a função utilidade no método multicritério SAW", conforme apresentada adiante.

$$
\text { Criticidade }_{i}=\sum_{j=1}^{n} p_{j} r_{i j}
$$

Em que,

Criticidade (ou função de utilidade no método SAW) do i-ésimo componente para i=1,2,..., n: expresso por um número Real

pj: é o peso do j-ésimo critério, sendo j=1, 2, ..,n, expresso por um número Real;

$r_{i j}$ : é o dado que representa a relação do i-ésimo componente com o j-ésimo critério, expresso por um número Real.

\section{Resultados}

O modelo matemático é utilizado para se determinar a criticidade dos elementos que compõem o sistema complexo MagLev-Cobra. Os resultados obtidos para cada componente são, hierarquizados pelos maiores Índices de Criticidade, conforme apresentado na Tabela 2 que expõe os 10 mais críticos e os 10 menos críticos. 
Tabela 2 - Lista hierarquizada por índice de criticidade dos componentes do sistema MagLevCobra

\begin{tabular}{|c|c|c|}
\hline Ranking & Componentes & Índice de Risco (IR) \\
\hline 10 & Criostato & 188,5 \\
\hline 20 & Primário do motor linear & 150,5 \\
\hline 30 & Folha de porta & 145,83 \\
\hline 40 & Fixação do criostato & 139,83 \\
\hline 50 & Pistão direito & 137,83 \\
\hline 60 & Estrutura de fixação das rodas de segurança & 135,67 \\
\hline 70 & Inversor de frequência & 135,67 \\
\hline 80 & Bomba hidráulica & 135,67 \\
\hline 90 & Estrutura de fixação dos Criostatos & 135,5 \\
\hline 100 & Pistão esquerdo & 133,83 \\
\hline 440 & Vedação entre vagões & 85,33 \\
\hline 450 & Sensor de altura & 83,83 \\
\hline 460 & Sensor de contagem de barras & 81,83 \\
\hline 470 & Pega mão & 81,33 \\
\hline 480 & $H D$ & 74,33 \\
\hline 490 & Software de Proc. Dados & 73,83 \\
\hline 50 은 & Acelerômetro & 71,5 \\
\hline 510 & Medidor de freq. Do inversor & 71,5 \\
\hline $52^{\circ}$ & Amperímetro & 61,5 \\
\hline 530 & Voltímetro & 61,5 \\
\hline
\end{tabular}

\section{Discussões}

Por meio da avaliação dessa lista verificou-se que o Criostato, um dos componentes responsáveis pela levitação do MagLev-Cobra, é o elemento mais crítico do sistema, apresentando um IR de 188,50 , seguido do primário do motor linear $(150,50)$, folha de porta $(145,83)$, fixação do criostato $(139,83)$, pistão direito $(137,83)$ e estrutura de fixação das rodas de segurança $(135,67)$.

Dessa forma, percebe-se que os dois componentes que são diretamente ligados ao sistema de levitação, Criostato e Fixação do Criostato, aparecem na primeira e quarta colocações, respectivamente. Por esse motivo, esses componentes, além dos demais citados, são aqueles que necessitam de maior atenção por parte do Planejamento e Controle da Manutenção, seja com manutenções preventivas ou com utilização de sensores, pois, em caso de falha, geram um maior impacto na principal função do sistema, que é o transporte.

Os resultados expostos também podem apoiar a distribuição de recursos de custeio e capital, haja vista que o direcionamento se dará pela influência de cada componente na função principal do sistema complexo. 
Em contrapartida, os componentes menos críticos são o amperímetro e o voltímetro, que fazem parte do sistema de monitoramento, ambos apresentando um IR no valor de 61,50. Foi percebido que, além desses, os elementos que compõem esse sistema tendem a apresentar índices de risco mais baixos, como é o caso do medidor de frequência do inversor, que aparece na antepenúltima colocação, com um IR de 71,50.

Verifica-se também que a alavanca de comando, que não é um componente de alta complexidade e pertence ao sistema de controle veicular, aparece em uma posição intermediária da lista, com um índice de risco de 106,33. Isso ocorreu, pois esse componente obteve avaliação consideravelmente negativa segundo o critério correlação (C), dentre outras avaliações desfavoráveis, o que significa que ele apresenta um alto grau de relação com a principal função do sistema e, em caso de falha, poderia afetá-la de forma significativa.

O estudo de Costa (2017), que visa identificar o elemento mais crítico do MagLev-Cobra para fins de certificação e adequação às Normas Internacionais de Segurança Ferroviária, também apontou o criostato como o componente com maior índice de risco do sistema, estando em concordância com o resultado obtido neste artigo.

Constatou-se com esse estudo quais são os componentes mais críticos do sistema complexo MagLev-Cobra e que, consequentemente, necessitam de maior atenção por parte da manutenção.

\section{Conclusões}

Este artigo propõe o desenvolvimento de um modelo matemático multicritério capaz de identificar os componentes mais críticos de um sistema complexo, com aplicação de estudo de caso no trem de levitação magnética desenvolvido pela COPPE/ UFRJ, o MagLev-Cobra.

A análise de criticidade é tratada com um método utilizado no planejamento que vem adquirindo vasta empregabilidade, pois visa a identificação, de forma antecipada, de eventuais falhas que possam ocorrer em um sistema, contribuindo para o aumento da sua produtividade e confiabilidade. Por se tratar de uma técnica que necessita da avaliação de múltiplas variáveis, pode ser utilizada com o auxílio de métodos de análise multicritério de apoio à decisão.

A seleção dos critérios utilizados no modelo foi realizada por intermédio da revisão bibliográfica, onde foram analisados 46 artigos, dos quais foi possível identificar 182 critérios. Após essa identificação foram selecionados 25 critérios que melhor se adequavam ao modelo matemático.

O método FSAW mostrou-se eficaz para o alcance dos objetivos deste artigo, uma vez que foi possível desenvolver o modelo matemático, aplicá-lo ao objeto do estudo de caso, o MagLev-Cobra, e identificar os índices de risco dos seus componentes, gerando uma lista hierarquizada dos mesmos. 
Após a modelagem concluiu-se que o criostato é o elemento mais crítico do sistema MagLevCobra, apresentando um índice de risco de 188,50. Tal resultado, incluído na lista hierarquizada apresentada na Tabela 2, foi apresentado aos integrantes do Projeto Maglev-Cobra que ratificaram a qualidade da resposta do modelo.

Considerando-se que os sistemas ditos complexos podem apresentar diferentes critérios para análise de risco, inclusive o MagLev-Cobra, o modelo matemático desenvolvido permite avaliar a criticidade dos elementos que compõe esses sistemas com maior precisão, visto que utiliza quantidade maior de critérios do que os modelos tradicionais, permitindo reconhecer quais são aqueles que oferecem um maior risco à sua principal função. Desse modo, é possível que o Planejamento e Controle de Manutenção se organize, priorizando esses componentes e antecipando-se às eventuais falhas que eles possam causar.

Esse processo resulta em um maior controle sobre o sistema, gerando um aumento da sua produtividade e da qualidade das atividades de manutenção, além de uma aplicação mais adequada dos recursos, que são limitados, evitando-se desperdícios e investimentos em áreas ou componentes de baixo impacto na função do sistema.

\section{Referências}

Andrade, M. R. S., \& Turrioni, J. B. (2015). Uma metodologia de análise dos aspectos e impactos ambientais através da utilização do FMEA. Recuperado em 04 agosto, 2017, de https://www.researchgate.net/profile/Joao_Turrioni/publication/267694135_UMA_METODOLOGIA _DE_ANALISE_DOS_ASPECTOS_E_IMPACTOS_AMBIENTAIS_ATRAVES_DA_UTILIZACAO_DO_FMEA/Ii nks/551aa5590cf2f51a6fea91cd.pdf.

Azizi, A., \& Fathi, K. (2014). Selection of optimum maintenance strategies based on a fuzzy analytic hierarchy process. Management Science Letters, 4(5), 893-898.

Baran, L. R. (2015). Proposta de um modelo multicritério para determinação da criticidade na gestão da manutenção industrial. Dissertação de mestrado, Universidade Tecnológica Federal do Paraná, Paraná, PR, Brasil. Disponível: http://repositorio.utfpr.edu.br/jspui/handle/1/1450.

Ben, F. (2006, outubro). Utilização do método AHP em decisões de investimento ambiental. Encontro Nacional de Engenharia de Produção, Fortaleza, CE, Brasil, 26.

Bowles, J. B., \& Peláez, C. E. (1995, June). Fuzzy logic prioritization of failures in a system failure mode, effects and criticality analysis. Reliability Engineering and System Safety, 50, 203-213.

British Standard Institution (1991). BS 5760: Reliability of Systems, Equipment and Components-Part 5. Guide to Failure Modes, Effects and Criticality Analysis (FMEA and FMECA) [Manual]. United Kingdom: British Standard Institution.

Brum, D. S., Do Carmo, S.C., \& Moreira, T.C. (2017). Modelo matemático Fuzzy-multicritério para avaliar desempenho de tripulações de rebocadores. Trabalho de Conclusão do Curso, Universidade Estácio de Sá, Rio de Janeiro, RJ, Brasil. 
Bueno, P. M. S., Ikuno, F. S., De Araújo, A. S., Lima, J. N. O., Moreira, J. R. M., \& De Melo, L. A. V. (2015). Uma iniciativa para aprimorar a gestão de riscos de segurança da informação na administração pública federal. Recuperado em 3 agosto, 2017, de https://siaiap34.univali.br/sbseg2015/anais/WRAC/artigoWRAC01.pdf.

Cavalcante, C. A. V., \& De Almeida, A.T. (2005). Modelo multicritério de apoio a decisão para o planejamento de manutenção preventiva utilizando PROMETHEE II em situações de incerteza. Pesquisa Operacional, 25(2). Recuperado em 10 agosto, 2017, de http://dx.doi.org/10.1590/S010174382005000200007.

Cavalcante, C.A.V., \& De Almeida, A.T. (2007). A multi-criteria decision-aiding model using PROMETHEE III for preventive maintenance planning under uncertain conditions. Journal of Quality in Maintenance Engineering, 13(4), 385-397. Retrieved May 17, 2017, from https://doi.org/10.1108/13552510710829470.

Cavalcante, C. A. V., Ferreira, R. J. P, Brito, A. J. M., \& De Almeida, A.T. (2007, agosto). Modelo multicritério para priorização de causas de falha: uma crítica ao FMECA. Simpósio Brasileiro de Pesquisa Operacional, Fortaleza, CE, Brasil, 39.

Chareonsuk, C., Nagarur, N., \& Tabucanon, M. T. (1997, March). A multicriteria approach to the selection of preventive maintenance intervals. International Journal Production Economics, 49, pp.55-64.

Chou, S.Y., Chang, Y. H., \& Shen, C.Y. (2008, August 16). A fuzzy simple additive weighting system under group decision-making for facility location selection with objective/subjective attributes. European Journal of Operational Research, 189(1), 132-145.

Costa, F. S. (2017). Adequação do Projeto Maglev Cobra às Normas Internacionais de Segurança Ferroviária com Análise Aplicada aos Levitadores do Veículo. Dissertação de Mestrado, Instituto Alberto Luiz Coimbra de Pós-graduação e Pesquisa de Engenharia - COPPE/UFRJ, Rio de Janeiro, RJ, Brasil.

Da Silva, A. C. S., \& Belderrain, M. C. N. (2009). O problema de seleção de fornecedores: abordagem AHP com uso de ratings. Simpósio Brasileiro de Pesquisa Operacional, Rio Grande do Sul, RS, Brasil, 42.

De Abreu, L. M., Granemann, S. R., Gartner, I., \& Bernardes, R. S. (2000). Escolha de um programa de controle da qualidade da água para consumo humano: aplicação do Método AHP. Revista Brasileira de Engenharia Agrícola e Ambiental, 4(2), 257-262.

De Almeida, A.T. (2001). Multicriteria decision making on maintenance: Spares and contracts planning. European Journal of Operational Research, 129(2), 235-241.

De Almeida, A. T. (2005). Modelagem multicritério para seleção de intervalos de manutenção preventiva baseada na teoria da utilidade multiatributo. Pesquisa Operacional, 25(1), 69-81.

De Almeida, L. N., Moré, J. D., \& Villela, L. E. (2010). Avaliação Fuzzy da percepção de empresários do APL de moda intima de Nova Friburgo sobre o desempenho em relação à sustentabilidade ambiental. Recuperado em 5 setembro, 2017, de http://www.sucena.eng.br/eng_producao/2015/2015_1_m\%C3\%A9dia\%20fuzzy\%20triangular\%20 2\%20Jesus\%20Detalhado.pdf. 
De Oliveira, E. C. B., Alencar, L. H., \& Costa, A. P. C. S. (2012). Modelo para classificação de projetos baseado no apoio multicritério a decisão. Congresso Latino-lberoamericano de Investigación Operativa Simpósio Brasileiro de Pesquisa Operacional, Rio de Janeiro, RJ, Brasil, 1.

Department of Defense - United States of America. (1980). Military-Standard-1629A: Procedures for performing a failure mode, Effects and Criticality Analysis [Manual]. Washington, DC: Department of Defense - United States of America.

Department of Defense - United States of America. (2012). Military-Stdandart-882E: System Safety [Manual]. Washington, DC: Department of Defense - United States of America.

Ferraboli, R., Jr. (2006). Exercício real de decisão com a aplicação do método Analytic Hierarchy Process (AHP). Revista Integração, 12(46), 263-272.

Helmann, K. S., \& Marçal, R. F. M. (2007). Método multicritério de apoio à decisão na gestão da manutenção: aplicação do método ELECTRE I na seleção de equipamentos críticos para processo. Revista Gestão Industrial, 3(1), 123-133.

Hijes, F. C. G. L., \& Cartagena, J. J. R. (2006). Maintenance strategy based on a multicriterion classification of equipments. Reliability Engineering and System Safety, 91(4), 444-451.

Ilangkumaran, M., \& Kumanan, S. (2009). Selection of maintenance policy for textile industry using hybrid multi-criteria decision making approach. Journal of Manufacturing Technology Management, 20(7), 1009-1022.

Jaderi, F., Ibrahim, Z. Z., Jaafarzadeh, N., Abdullah, R., Yavari, A. R., Shamsudin, M. N., \& Nabavi, S. M. B. (2014). Criticality Analysis Using Risk Assessment-Based Maintenance of a Petrochemical Company. Polish Journal Environmental Studies, 23(6), 2033-2037.

Leal, I. C., Jr. (2010). Método de escolha modal para transporte de produtos perigosos com base em medidas de ecoeficiência. Tese de doutorado, Instituto Alberto Luiz Coimbra de Pós-Graduação e Pesquisa de Engenharia, Rio de Janeiro, RJ, Brasil.

Leal, F., De Pinho, A. F., \& De Almeida, D. A. (2006). Análise de falhas através da aplicação do FMEA e da teoria Grey. Revista Gestão Industrial, 2(1), 78-88.

Lins, F. E. (2009). Modelo multicritério para priorização de equipamentos hospitalares para manutenção programada. Dissertação de mestrado, Universidade Federal de Pernambuco, Pernambuco, PE, Brasil.

Lins, P. H. C., Garcez, T. V., \& De Almeida, A. T. (2011). Análise multicritério da criticidade de modos de falha definidos com o auxílio da abordagem MCC. Simpósio Brasileiro de Pesquisa Operacional, Ubatuba, SP, Brasil, 43.

Macedo, M. A. S. (2011). Contribuição metodológica para a determinação da criticidade de equipamentos na gestão da manutenção. Tese de mestrado, Universidade Tecnológica Federal do Paraná, Ponta Grossa, PR, Brasil.

Marins, C. S., Souza, D. O., \& Barros, M. S. (2009). O uso do método de Análise Hierárquica (AHP) na tomada de decisões gerenciais - Um estudo de caso. Simpósio Brasileiro de Pesquisa Operacional, Porto Seguro, BA, Brasil, 41. 
Mierau, D. J. (n.d.). Defining Holistic Asset Criticality to Manage Risk. Pharmaceutical Engineering Magazine. Retrieved August 21, 2017, from https://www.Ice.com/Defining-Holistic-Asset-Criticalityto-Manage-Risk-1414.html.

MITRE Corporate Communications and Public Affairs. (2014). Systems Engineering Guide: Risk Impact Assessment and Prioritization [Manual]. Bedford, MA: The MITRE Corporation.

Montevechi, J. A. B., \& De Pinho, A. F. (n.d.). Incertezas em algoritmos de programação de operações. Recuperado em 8 setembro, 2017, de http://www.abepro.org.br/biblioteca/ENEGEP1999_A0038.PDF.

Moreira, M. P. (2009). Priorização dos modos de falha de equipamentos utilizando os métodos de análise multicritério PROMETHEE e FUZZY PROMETHEE. Dissertação de mestrado, Pontífica Universidade Católica do Rio de Janeiro, Rio de Janeiro, RJ, Brasil.

Moteff, J. (2005). Risk Management and Critical Infrastructure Protection: Assessing, Integrating, and Managing Threats, Vulnerabilities and Consequences. Report for Congress, Document RL32561, Congressional Research Service, Library of Congress, Washington, DC, USA.

Nakajima, G. T., Da Silva, F. C., \& Miranda, J. I. (2007). Avaliação de processos de compostagem em URC's paulistas pela análise multicritério. International Conference on Engineering and Computer Education, São Paulo, SP, Brasil, 5.

Noronha, S. M. D. (1998). Um modelo multicritérios para apoiar a decisão da escolha do combustível para alimentação de caldeiras usadas na indústria têxtil. Dissertação de mestrado, Universidade Federal de Santa Catarina, Florianópolis, SC, Brasil.

Olsen, C. (2015). Development and Execution of Asset Criticality Framework: A Study of Water and Wastewater Infrastructure at Toowoomba Regional Council. Dissertation for Bachelor of Civil Engineering, University of Southern Queensland, Queensland, Australia. Disponível:

https://eprints.usq.edu.au/29291/.

Pamplona, E. O. (1999). Avaliação qualitativa de cost drivers pelo método AHP. Anais do Congresso Brasileiro de Custos, São Paulo, SP, Brasil, 6.

Prado, A. A. A., Souza, K., \& Yoshizaki, H. (2009). Seleção de fornecedores de transporte utilizando análise de decisão multi-critério. Recuperado em 20 Julho, 2017, de https://www.researchgate.net/profile/Hugo_Yoshizaki/publication/228519894_SELECAO_DE_FORN ECEDORES_DE_TRANSPORTE_UTILIZANDO_ANALISE_DE_DECISAO_MULTI-

CRITERIO/links/00b49527b63b63c538000000/SELECAO-DE-FORNECEDORES-DE-TRANSPORTEUTILIZANDO-ANALISE-DE-DECISAO-MULTI-CRITERIO.pdf.

Rossoni, C., \& Meireles, M. (2011, agosto). Decisão multicritério: Uma análise dos resultados obtidos pelos métodos T-ODA e AHP. Simpósio de Administração da Produção, Logística e Operações Internacionais - SIMPOI, Piracicaba, SP, Brasil, 14.

Sachdeva, A., Kumar, D., \& Kumar, P. (2009, January). Multi-factor failure mode critically analysis using TOPSIS. Journal of Industrial Engineering International, 8, pp. 1-9.

Sardinha, G. P., Claro, F. A. E., \& Pereira, R. L. (2009). Uso combinado do AHP e do FMEA para análise de riscos em gerenciamento de projetos. Recuperado em 21 julho, 2017, de https://www.aedb.br/seget/arquivos/artigos09/548_SCP_SEGeT_reedit021009.pdf. 
Secretaria de Logística e Tecnologia da Informação; Centro de Tecnologia da Informação Renato Archer do Ministério da Ciência, Tecnologia e Inovação; Fundação de Apoio à Capacitação em Tecnologia da Informação. (2015). Metodologia de gestão de riscos de segurança da informação: Desenvolvimento de metodologia e ferramenta de software público de arquitetura aberta para gestão de riscos de segurança da informação na Administração Pública Federal (Relatório RM2/2015), Brasília, DF, Fundação de Apoio à Capacitação em TI.

Shaghaghia, M., \& Rezaieb, K. (2012, February). Failure Mode and Effects Analysis Using Generalized Mixture Operators. Journal of Optimization in Industrial Engineering, 11, pp. 1-10.

Sheng-Hsien, G. T., \& Shin-Yann, M. H. (1996). Failure mode and effects analysis: An integrated approach for product design and process control. International Journal of Quality \& Reliability Management, 13, pp. 8-26.

Sucena, M. P. (2002). Subsídios para a alocação de recursos financeiros em sistemas de transportes urbanos sobre trilhos baseado em critérios técnicos. Dissertação de mestrado, Instituto Militar de Engenharia - IME, Rio de Janeiro, RJ, Brasil.

Sucena, M. P. (2017). Apontamentos da disciplina Métodos Matemáticos Aplicados a Engenharia de Produção [Apostila de Métodos Matemáticos Aplicados à Engenharia de Produção]. Rio de Janeiro: UNESA.

Szajubok, N. K., Mota, C. M. M., \& De Almeida, A. T. (2006). Uso do método multicritério electre tri para classificação de estoques na construção civil. Pesquisa Operacional, 26(3), 625-648. Recuperado em 20 junho, 2017 de http://www.scielo.br/scielo.php?pid=S0101$74382006000300010 \&$ script=sci_arttext\&tIng=es.

Xu, K., Tang, L.C., Xie, M., HO, S.L., \& Zhu, M.L. (2002, January). Fuzzy assessment of FMEA for engine systems. Reliability Engineering and System Safety, 75, pp. 17-19.

Zammori, F., \& Gabbrielli, R. (2012, February). ANP/RPN: A Multi Criteria Evaluation of the Risk Priority Number. Quality and Reliability Engineering International, 28(1), pp. 85-104. 